\title{
Effect of carbohydrate mouth rinse on resistance training performance in trained men
}

\author{
- Christian Wisdom Magtajas Valleser, ๑Adrian Gabriel Rivera
}

Department of Sports Science, College of Human Kinetics, University of the Philippines Diliman, Quezon City, Philippines.

\begin{abstract}
The effect of carbohydrate (CHO) mouth rinse as an ergogenic aid in aerobic activity is wellestablished. However, its effect on short-duration, high-intensity resistance training is yet to be explored. This study aimed to investigate the effect of a $\mathrm{CHO}$ mouth rinse on resistance training performance of trained men in terms of total training volume and perceived exertion in a randomized, cross-over, double-blind design. Fourteen trained men participated in three repeated experimental resistance exercise sessions. Each resistance exercise session consisted of three sets performed until volitional fatigue for the deadlift, squat, bench press, and military press with a load $75 \%$ of their tested 1-repetition maximum and 2 minutes rest interval between sets. At the start of each experimental session, and immediately before the third exercise in the sequence, subjects were given a $100 \mathrm{ml}$ solution of either $\mathrm{CHO}$, artificial sweetener (placebo), or water (control) as a mouth rinse for 10 seconds. Comparisons were evaluated with a repeated-measures analysis of variance at $\alpha=0.05$. A CHO mouth rinse significantly increased total training volume compared to both the placebo $(+23.1 \%)$ and control $(+25.9 \%)$. The effect on perceived exertion was similar for the $\mathrm{CHO}$ solution and the non-calorie sweetener solution. The authors conclude that a carbohydrate mouth rinse may benefit resistance training performance in terms of total training volume and perceived exertion in trained men.
\end{abstract}

Keywords. Carbohydrate mouth rinse, resistance training, training volume.

\section{Introduction}

Ergogenic aids provide performance enhancement to help athletes reach their peak form. Carbohydrate $(\mathrm{CHO})$ ingestion before and during exercise is one of the most well researched and proven ergogenic aids (Thomas et al., 2016). However, the mechanisms behind its ergogenic effect on short durations (activities $<60$ mins in duration) are inconclusive. Numerous studies claim that $\mathrm{CHO}$ ingestion improves performance in exercise that last less than 60 minutes (Haff et al., 1999; Haff et al., 2001; Wax et al., 2012). The mere presence of $\mathrm{CHO}$ in the oral cavity may have an ergogenic effect as backed by research that shows a carbohydrate mouth rinse may be a viable option for high-intensity exercise, such as resistance training, that lasts less than an hour (Jeukendrup, 2014). Resistance training has been shown to improve muscular hypertrophy, strength, and power (Schoenfeld et al., 2017). To achieve these muscular improvements total training volume should be increased as training progresses (Schoenfeld et al., 2019). Since CHO mouth rinsing has been shown to reduce fatigue in weight training (Jensen et al., 2015), this shows that oral rinsing of $\mathrm{CHO}$ may help improve total training volume and thus increase muscular fitness.

Most studies on $\mathrm{CHO}$ mouth rinsing are on cycle time trials (Carter et al., 2004; Fraga et al., 2017; Pomportes et al., 2017) while we found only one that measures its direct effect on weight training (Decimoni et al., 2018). A CHO mouth rinse solution produced positive effects on the studies done on cycle time trials and weight training while its effect on the Rating of Perceived Exertion (RPE) remains inconclusive. Thus, the aim of this study was to determine the effect of $\mathrm{CHO}$ mouth rinse on resistance training performance in terms of total training volume and perceived exertion. 


\section{Methods}

\section{Research Design}

This study is a randomized, cross-over, doubleblind design wherein 14 trained men performed three different experimental resistance exercise sessions to examine the effect of a $\mathrm{CHO}$ mouth rinse on resistance training performance.

\section{Subjects and Sampling}

The research subjects were selected based on the following: (1) male, (2) 18 to 22 years old, (3) at least one but no more than two years of weight training experience, and (3) does not participate in any competitive sport. Subjects were acquired through volunteer sampling. A total of 14 trained men (Mage $20.13 \pm 0.83$ years) participated in the study. They had a mean training experience of 1.4 \pm 0.4 years. Subjects signed an informed consent form and were allowed to withdraw participation at any point. The paper was conducted according to the ethical guidelines in the Declaration of Helsinki and was approved by the research proposal panel including ethical considerations on 1 September 2019.

\section{Procedure}

The experiment was carried out over four sessions comprising of one day for estimated 1-Repetition Maximum (1-RM) testing and three days for the experimental trials. Testing sessions were separated by 72 hours for adequate rest between sessions. Each session began with five minutes of general warm-up followed by specific warm-up sets. During the first day, all participants were tested for their estimated 1-RM for the deadlift, squat, bench press, and military press. Each resistance exercise session consisted of three sets of four exercises that were performed until volitional fatigue in the deadlift, squat, bench press, and military press, with a 10-RM load (derived as $75 \%$ of their 1-RM based one the National Strength and Conditioning Association load chart) and two minutes of rest intervals between sets. During the next three testing days, each participant randomly went through one of three conditions - a $\mathrm{CHO}$ (white sugar) mouth rinse, a non-calorie sweetener (placebo) mouth rinse, or water (control) mouth rinse.

At the start of each experimental session, and immediately before the third exercise in the sequence, subjects were given a $100 \mathrm{ml}$ solution of either $\mathrm{CHO}$, non-calorie sweetener, or water. The subjects were instructed to rinse the fluid around their mouths for 10 seconds and then spit the solution into a container. They received the treatments twice during each session - right before the start of the first exercise and right before the start of the third exercise. Thus, the mouth rinse was applied before the start and in the middle of each session. The participants were not allowed to hydrate or drink during the entire session. After each set of an exercise, participants were asked to rate their perceived exertion in a 1- to 10-point Borg RPE scale. The Borg RPE is a method of measuring perceived physical activity intensity based on physical sensations like increased heart rate, increased respiration rate, increased sweating, and muscular fatigue (Borg, 1998).

\section{Statistical Analysis}

Descriptive data were presented as mean \pm standard deviation. Total training volume (sets $\mathrm{x}$ repetitions $\mathrm{x}$ load) and average RPE between testing conditions are compared with repeated measures analysis of variance (ANOVA). The normality of distribution was established through the Shapiro-Wilk test. Significant results in the repeated measures ANOVA were followed by Tukey's HSD Post-Hoc test. Statistical significance was set a priori at $\alpha=0.05$.

\section{Results}

\section{Total Training Volume}

There is a significant difference in total training volume (CHO, placebo, control) based on the repeated measures ANOVA $(p<0.01)$ as presented in Table 1. Tukey's HSD post hoc pointed out a significant difference in favor of a 
$\mathrm{CHO}$ mouth rinse over both water $(25.9 \%$ difference, $\mathrm{p}<0.01$ ) and a non-calorie sweetener (23.1\% difference, $\mathrm{p}<0.01$ ). Furthermore, there was no difference between water and the noncalorie sweetener (2.3\% difference) mouth rinse solutions. These findings suggest that a $\mathrm{CHO}$ mouth rinse can be effective in acutely improving resistance training performance via increasing total training volume.

\section{Table 1}

Total training volume in the training sessions with a carbohydrate, placebo, and control mouth rinse.

\begin{tabular}{lccc}
\hline Treatment & Mean & SD & P \\
\hline Carbohydrate & 8884 & 3380 & \\
Placebo & 7219 & 2829 & $<0.01$ \\
Control & 7054 & 2755 & \\
\hline
\end{tabular}

Table 2

Average RPE in the training sessions with a carbohydrate, placebo, and control mouth rinse.

\begin{tabular}{lccc}
\hline Treatment & Mean & SD & P \\
\hline Carbohydrate & 8.0 & 1.1 & 0.02 \\
Placebo & 8.6 & 0.8 & \\
Control & 8.3 & 0.8 & \\
\hline
\end{tabular}

\section{Average RPE}

There is a significant difference in average RPE observed from the repeated measures ANOVA ( $p$ $=0.02$ ) as summarized in Table 2 . The following Tukey's Post-Hoc HSD indicates a significant difference in RPE only between the training sessions with a $\mathrm{CHO}$ mouth rinse and water $(6.3 \%$ difference, $p<0.05)$. There was no significant difference in RPE between water and a non-calorie sweetener mouth rinse $(2.8 \%$ difference). Interestingly, there was also no significant difference in RPE between a $\mathrm{CHO}$ mouth rinse and a non-calorie sweetener mouth rinse $(4.1 \%$ difference). This suggests that although a noncalorie sweetener mouth rinse does not have the same magnitude effect on total training volume as a $\mathrm{CHO}$ mouth rinse, it does have a comparable effect on perceived exertion.

\section{Discussion}

The findings in this study indicate that a $\mathrm{CHO}$ mouth rinse results in significantly higher total training volume when compared to a non-calorie sweetener solution or just plain water. This affirms previous reports by Decimoni et al. (2018) and Jeukendrup (2018) who found that $\mathrm{CHO}$ mouth rinses work better in high intensity, shortduration exercises. RPE also improved with a $\mathrm{CHO}$ mouth rinse over plain water. This effect corroborates the findings of Decimoni et al. (2018) and Pomportes at al. (2017). Jeukendrup (2010) indicates that this is due to a central mechanism in the oral cavity that is activated by the presence of $\mathrm{CHO}$. This also supports the functional magnetic resonance imaging observations by Carter at al. (2004) which associated a greater activation of the dopaminergic system of the ventral striatum with the presence of $\mathrm{CHO}$ which may result in higher arousal, motivation, motor control and attenuation of fatigue (Chambers et al., 2009; Jensen et al., 2015). This may also explain why the effects of $\mathrm{CHO}$ are only commonly seen in high intensity, short-duration exercises as there may be a minimum threshold of cumulative fatiguing stimuli before significant effects can be detected. These findings contradict the observations of previous studies including those of Witham \& McKinney (2007), Beelen et al. (2009), and Painelli et al. (2011). Haff et al. (2001) propose that the varying results draw a clear contrast in acute effects according to the duration and intensity in these studies. A trend in favor of $\mathrm{CHO}$ is observed in high intensity, short-duration activities.

The effect on RPE with a non-calorie sweetener solution is interestingly similar to a $\mathrm{CHO}$ solution. This contradicts previous studies (Hawkins et al., 2017; Jeukendrup, 2014) that concluded sweetness alone is not enough to elicit an ergogenic response similar to $\mathrm{CHO}$. Although several studies have pointed toward $\mathrm{CHO}$ mouth rinsing increasing exercise performance with no effect on RPE (Carter et al., 2004; Chambers et al., 2009; Fraga et 
al., 2017), a common trend is that these studies employed a cycle time trial wherein subjects would generally pace themselves throughout the duration of the exercise (Clarke et al, 2017). In contrast, this study required subjects to do every set to volitional fatigue or maximum muscular effort. Performing maximal effort produces a more observable difference in perception of effort (Decimoni et al., 2018). A non-calorie sweetener solution showed the same effect on perceived exertion, and thus fatigue, as $\mathrm{CHO}$ but without the same ergogenic effect on total training volume. Although this may suggest that a non-calorie sweetener solution elicits the same fatigue attenuation effect that $\mathrm{CHO}$ has, further investigation is needed regarding this observation. Furthermore, reducing the perception of effort without improving resistance training performance provides little to no practical application for a non-calorie sweetener mouth rinse.

The limitations of the study include its applicability to different groups such as beginners and highly experienced individuals. Furthermore, the diet and caloric intake of the subjects were not regulated. This study focused on trained men with more than one but not over two years of weight training experience and have not participated in any competitive sport during those years. All 4 exercises were done until volitional fatigue. As such, individual tolerances of fatigue may vary as measured using a Borg RPE scale which is a subjective form of evaluation. The same amount of $\mathrm{CHO}$ and placebo solutions were given to all participants. Lastly, the long-term effects of $\mathrm{CHO}$ mouth rinsing in terms of adaptations and progress in resistance training cannot be established in this study.

\section{Acknowledgments}

The authors appreciate the College of Human Kinetics, University of the Philippines Diliman for allowing us to use their facilities to conduct this research.

\section{Funding}

No external funding was received for this study.

\section{Conflict of Interest}

The authors declare no conflicts of interest.

\section{References}

Beelen, M., Berghuis, J., Bonaparte, B., Ballak, S.B., Jeukendrup, A.E., \& van Loon, L.J.C. (2009). Carbohydrate mouth rinsing in the fed state: lack of enhancement of time-trial performance. Int J Sport Nutr Exerc Metab, 19(4), 400-409.

Borg, G. (1998). Borg's perceived exertion and pain scales. Champaign, IL: Human Kinetics.

Carter, J.M., Jeukendrup, A.E., \& Jones, D.A. (2004). The effect of carbohydrate mouth rinse on 1h cycle time trial performance. Med Sci Sports Exerc, 36(12), 2107-2111.

Chambers, E.S., Bridge, M.W., \& Jones, D.A. (2009). Carbohydrate sensing in the human mouth: effects on exercise performance and brain activity. $J$ Physiol, 587(8), 1779-1794.

Clarke, N.D., Hammond, S., Kornilios, E., \& Mundy, P.D. (2017). Carbohydrate mouth rinse improves morning high intensity exercise performance. Eur $\mathrm{J}$ Sport Sci, 17(8), 955-963.

Decimoni, L.S., Curty, V.M., Almeida, L., Koch, A.J., Willardson, J.M., \& Machado, M. (2018). Carbohydrate mouth rinsing improves resistance training session performance. Int $J$ Sports Sci Coach, 13(5), 804-809.

Fraga, C., Velasques, B., Koch, A.J., Machado, M., Paulucio, D., Ribeiro, P., \& Pompeu, F.A.M.S. (2017). Carbohydrate mouth rinse enhances time to exhaustion during treadmill exercise. Clin Physiol Funct Imaging, 37(1), 17-22.

Haff, G.G., Schroeder, C.A., Koch, A.J., Kuphal, K.E., Comeau, M.J., \& Potteiger, J.A. (2001). The effects of supplemental carbohydrate ingestion on intermittent isokinetic leg exercise. J Sport Med Phys Fit, 41(2), 216-222.

Haff, G.G., Stone, M.H., Warren, B.J., Keith, R., Johnson, R.L., Nieman, D.C., Williams, F., Kirksey, K.B. (1999). The effect of carbohydrate supplementation on multiple sessions and bouts of 
resistance exercise. J Strength Cond Res, 13(2), 111117.

Hawkins, K.R., Krishnan, S., Ringos, L., Garcia, V., \& Cooper, J.A. (2017). Running performance with nutritive and non-nutritive sweetened mouth rinses. Int J Sport Physiol, 12(8), 1105-1110.

Jensen, M., Stellingwerff, T., \& Klimstra, M. (2015). Carbohydrate mouth rinse counters fatigue related strength reduction. Int J Sport Nutr Exe, 25(3), 252261.

Jeukendrup, A. (2014). A step towards personalized sports nutrition: carbohydrate intake during exercise. Sports Med, 44 (Suppl 1), 25-33.

Jeukendrup, A.E., \& Chambers, E.S. (2010). Oral carbohydrate sensing and exercise performance. Curr Opin Clin Nutr Metab Care, 13(4), 447-451.

Painelli, V.S., Roschel, H., Gualano, B., Del-Favero, S., Benatti, F.B., Ugrinowitsch, V.T., Tricoli, V., Lancha, A.H. (2011). The effect of carbohydrate mouth rinse on maximal strength and strength endurance. Eur J Appl Physiol, 111(9), 2381-2386.

Pomportes, L., Brisswalter, J., Casini, L., Hays, A., \& Davranche, K. (2017). Cognitive performance enhancement induced by caffeine, carbohydrate and guarana mouth rinsing during submaximal exercise.
Nutrients, 9(6), 589.

Schoenfeld, B.J., Contreras, B., Krieger, J., Grgic, J., Delcastillo, K., Belliard, R., \& Alto, A. (2019). Resistance training volume enhances muscle hypertrophy but not strength in trained men. Med Sci Sports Exerc, 54(1), 94-103.

Schoenfeld, B.J., Grgic, J., Ogborn, D., \& Krieger, J.W. (2017). Strength and hypertrophy adaptations between low- vs. high-load resistance training: A systematic review and meta-analysis.J Strength Cond Res, 31(12), 3508-3523.

Thomas, D.T., Erdman, K.A., \& Burke, L.M. (2016). Position of the academy of nutrition and dietetics, dietitians of Canada, and the American college of sports medicine: nutrition and athletic performance. J Acad Nutr Diet, 116(3), 501-528.

Wax, B., Brown, S.P., Webb, H.E., \& Kavazis, A.N. (2012). Effects of Carbohydrate supplementation on force output and time to exhaustion during static leg contractions superimposed with electromyostimulation. J Strength Cond Res, 26(6), 1717-1723.

Whitham, M. \& McKinney, J. (2007). Effect of a carbohydrate mouthwash on running time trial performance. J Sports Sci, 25(12), 1385-1392. 